In Fig. 1 the oriented spermatid contains the mitochondrial nebenkern $(N)$ at the posterior end of the nucleus, the proximal centriole lying in between. The distal centriole and the flagellum are at $(C)$ and the Golgi body at $(G)$. A further account of this work is being published elsewhere.

This work was performed in part under the auspices of the U.S. Atomic Energy Commission.

\section{J. Brontë Gatenby}

Argonne National Laboratory,

Lemont, Illinois.

\section{A. J. Dalton}

National Cancer Institute,

Bethesda, Maryland. July 21.

${ }^{2}$ Chatton, E., and Tuzet, O., C.R. Acad. Sci., Paris, 213 (1941).

\section{Synaptic Transmission in a Sodium-free Medium}

THE observation of Lorente de Nóx, that small frog axons ("Et fibres") are excitable in media where the sodium is totally replaced by certain quaternary ammonium ions, has since been extended to crustacean muscle fibres ${ }^{2}$, frog $A$-fibres ${ }^{3,4}$, frog spinal ganglion cell-bodies ${ }^{5}$ and mammalian striated muscle $e^{6,7}$ and nerve ${ }^{8}$ fibres. Such findings suggested that the membrane of muscle fibres and neurones in different species was capable of maintaining excitability when the external sodium was replaced by a favourable cation possessing a tetracovalent nitrogen atom. The purpose of the present study was to determine whether synaptic transmission could also be maintained in sodium-free media. It is well known that the membrane characteristics of the junctional region are somewhat different from those possessing the conductile activity, since the post-synaptic membrane produces graded responses by the action of the transmitter released from the pre-synaptic membrane. It was previously reported ${ }^{\theta}$ that in the frog spinal ganglion cell-bodies hydrazinium ion was the most favourable substitute for sodium ion; the action potentials of cell-bodies being very similar to those observed in normal Ringer. An attempt to replace sodium ion by hydrazinium ion was quite successful; the neuromuscular transmission was restored in the sodium-free hydrazinium solution.

Nerve sartorius preparations of frogs (Rana pipiens) were used. The sodium-free hydrazinium solution contained $2 \mathrm{~m} M$ potassium chloride, $1.8 \mathrm{~m} M$ calcium chloride with various amounts of hydrazine (Eastman) and sufficient sucrose to maintain isotonicity. Both the hydrazine base and the final solution were adjusted to $p H 6.5$ with hydrochloric acid.

Neuromuscular transmission of the nerve sartorius preparations was completely blocked when soaked in a sodium-free $224 \mathrm{~m} M$ sucrose solution (containing $2.0 \mathrm{~m} M$ potassium chloride and $1.8 \mathrm{~m} M$ calcium chloride) for 30-40 min. In addition, the individual muscle fibres lost their electrical excitability, and the sensitivity of the end-plate membrane to directly applied acetylcholine was greatly depressed as measured by the absence of any contraction upon the application of acetylcholine in concentrations less than $10^{-5} \mathrm{gm} . / \mathrm{ml}$. No end-plate potential could be recorded by applying nerve stimulations. The disappearance of the end-plate potential caused by lack of sodium is mainly due to a decrease in the amount of released acetylcholine rather than a decrease in the sensitivity of the end-plate membrane to it ${ }^{10}$. It was quite certain, therefore, that the activity of the terminal endings of motor nerve fibres disappeared in a sodium-free sucrose solution, although the residual electrical activity of nerve twigs innervating the sartorius muscle was usually still detectable.

A surprising restoration of neuromuscular transmission occurred in the preparations which were first immersed in sucrose for $60-90 \mathrm{~min}$. and then in a solution containing 80-100 $\mathrm{m} M$ hydrazine. Normal muscle contractions were induced by nerve stimulations about 10-20 min. after re-immersion in these solutions. Meanwhile, the resting potential of the muscle fibres maintained a normal value, and propagated muscle action potentials, similar to those observed in normal Ringer, were produced by intracellular direct stimulations. However, the values of the threshold of muscle fibres were considerably larger than those obtained in normal Ringer. With nerve stimulations the end-plate potentials could be recorded from the end-plate regions, and the propagated muscle spikes were observed from muscle fibres. The muscle contractions, however, disappeared shortly after continuous nerve stimulations (1/sec.) were applied for $10-20 \mathrm{~min}$., although the electrical activity of the nerve twigs innervating the sartorius muscle and the sensitivity of the end-plate membrane were maintained (visible muscle contractions were produced by direct applications of acetylcholine in concentrations of $10^{-7}-10^{-8} \mathrm{gm} . / \mathrm{ml}$.).

These experimental results demonstrated that, in addition to the activity of the motor nerve axon ${ }^{4}$, the activity of the muscle fibres with the post-synaptic membrane and of the motor terminal endings with the pre-synaptic membrane were also maintained in the sodium-free hydrazinium solution. Although a trace of sodium may be retained around the junctional regions, it is not likely that the concentration of sodium was sufficient to maintain transmission ${ }^{10}$. It seems as if the hydrazinium ion is acting in exactly the same way as the sodium ion does upon the postand pre-synaptic membrane. The experimental evidence ${ }^{11,12}$ obtained from spinal ganglion cellbodies, however, seems to exclude this possibility. Recently, synaptic transmission in the sympathetic ganglion of frogs has also been found to be maintained in a sodium-free hydrazinium solution.

This work was supported by U.S. Public Health Service Grant $B-1650$.

Research Laboratories, Department of Psychiatry,

University of Illinois College of Medicine, Chicago.

June 30.

1 Lorente de Nó, R., J. Cell Comp. Physiol., 33, Supp. 1 (1949).

${ }^{2}$ Fatt, P., and Katz, B., J. Physiol., 120, 171 (1953).

${ }^{3}$ Larramendi, L. M. H., Lorente de No, R., and Vidal, F., Nature, 178, 316 (1956).

${ }^{4}$ Lorente de Nó, R., Vidal, F., and Larramendi, L. M. H., Nature, 179, 737 (1957).

'Koketsu, K., Cerf, J. A., and Nishi, S., Nature, 181, 703 (1958).

Daniel, E. E., and Singh, H., Fed. Proc., 17, 362 (1958).

${ }^{7}$ Burnstork, G., and Straub, R. W., J. Physiol., 140, 156 (1958).

${ }^{8}$ Sugaya, E., and Laget, P., J. de Physiol., 50, 529 (1958).

- Koketsu, K., Cerf, J. A., and Nishi, S., Nature, 181, 1798 (1958). ${ }^{10}$ Fatt, P., and Katz, B., J. Physiol., 118, 73 (1952).

${ }^{11}$ Koketsu, K., Cerf, J. A., and Nishi, S., Fed. Proc., 17, 89 (1958).

${ }^{12}$ Koketsu, K., Cerf, J. A., and Nishi, S., J. Neurophysiol. (in the press). 